Revista de Matemática: Teoría y Aplicaciones 2009 16(2): 221-230

CIMPA - UCR ISSN: 1409-2433

\title{
A MIMETIC FINITE DIFFERENCE METHOD USING CRANK-NICOLSON SCHEME FOR UNSTEADY DIFFUSION EQUATION
}

\author{
ILIANA A. MANNARINO S.*
}

Recibido/Received: 20 Feb 2008 - Aceptado/Accepted: 8 Oct 2008

\begin{abstract}
In this article a new mimetic finite difference method to solve unsteady diffusion equation is presented. It uses Crank-Nicolson scheme to obtain time approximations and second order mimetic discretizations for gradient and divergence operators in space. The convergence of this new method is analyzed using Lax-Friedrichs equivalence theorem. This analysis is developed for one dimensional case. In addition to the analytical work, we provide experimental evidences that mimetic Crank-Nicolson scheme is better than standard finite difference because it achieves quadratic convergence rates, second order truncation errors and better approximations to the exact solution.
\end{abstract}

Keywords: mimetic scheme, finite difference method, unsteady diffusion equation, Lax-Friedrichs equivalence theorem.

\section{Resumen}

En este artículo se presenta un nuevo método mimético de diferencias finitas para resolver la ecuación no estática de difusión. Éste usa el esquema de Crank-Nicholson para obtener aproximaciones en tiempo y discretizaciones miméticas de segundo orden, para los operadores gradiente y divergencia, en el espacio. La convergenica de este nuevo método es analizada usando el teorema de equivalencia de Lax-Friedrichs. Este análisis es desarrollado para el caso unidimensional. Además del estudio teórico, se dan pruebas prácticas que evidencian que el esquema mimético tipo Crank-Nicholson

*Escuela de Matemáticas, Facultad de Ciencias, Universidad Central de Venezuela. Apto. Postal 6228, Carmelitas 1010, Caracas, Venezuela. E-Mail: iliana.mannarino@gmail.com, iliana.mannarino@ciens.ucv.ve. 
es mejor que el esquema tradicional de diferencias finitas ya que arroja tasas de convergencia cuadráticas, errores de truncamiento de segundo orden y mejores aproximaciones a la solución exacta.

Palabras clave: método mimético, método de diferencias finitas, ecuación no estática de difusión, teorema de equivalencia de Lax-Friedrichs.

Mathematics Subject Classification: 65D25, 65G99, 65M06.

\section{Introduction}

The most simple way to solve partial differential equations is the use of efficient methods like finite difference methods [4]. They assume that the discretization of differential equation and boundary conditions are simultaneously valid at the extended boundary nodes but this is not completely correct. To avoid this problem mimetic methods have been developed. They are based on the discretization of continuum differential operators like gradient and divergence [1]; they verify the discrete versions of Green identity and they guaranty that the discretization of the boundary conditions and of the differential equation are compatible. Combination of second order mimetic discretization with Crank-Nicolson approach in time has not been reported in the contest of unsteady diffusion equation. In this article is presented this new approach. Its content has been distributed in the following way. In the next section we shortly describe the second order mimetic discretization for gradient and divergence operators. Next, we present the one dimensional transient diffusion equation and a new mimetic scheme for its solution. After that, a convergence analysis is presented. Next, illustrative 1D test problem is developed. It provides a solid evidence of the advantage of new scheme against standard finite difference and finally we summarize the conclusions of the present work.

\section{Mimetic operators}

Mimetic methods $[1,3]$ should provide discretizations $[\mathbf{G}],[\mathbf{D}]$ and $[\mathbf{B}]$ for corresponding continuous gradient $(\nabla)$, divergence $(\nabla \cdot)$ and boundary operator $\left(\frac{\partial u}{\partial \vec{n}}\right)$. They satisfy a discrete version of Green identity:

$$
\langle D v, f\rangle_{Q}+\langle v, G f\rangle_{P}=\langle B, v f\rangle_{Q},
$$

where $P$ and $Q$ are inner product's weights. The operator $[\mathbf{B}]$ is obtained by the explicit formula:

$$
[\mathbf{B}]=Q[\mathbf{D}]+[\mathbf{G}]^{t} P
$$

and it is a very important operator for boundary conditions.

In order to obtain the description of operators $[\mathbf{G}]$ and $[\mathbf{D}]$, one dimensional mimetic grid is sketched in Figure 1.

In figure $1, x_{i}$ for $i=1, \ldots, N$ represented by vertical lines defines blocks edges, black inner points represent nodes. A peculiar feature of mimetic grid is that nodes and edges 


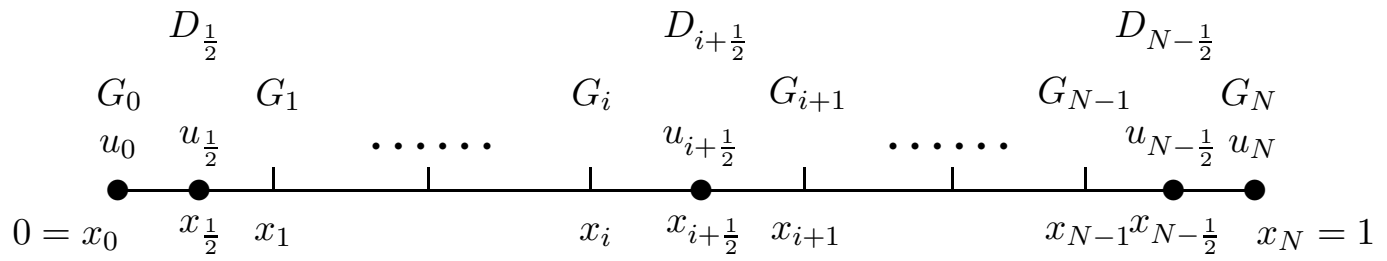

Figure 1: 1-D mimetic grid.

agree in boundaries. For simplicity, we assume that $x_{0}=0$ and $x_{N}=1$ and $h=\frac{1}{N}$. The sequence $u_{i}$ represents the solution of diffusion equation. The discrete form of the gradient [G] operator at inner edges $x_{i}$ is given by:

$$
(G u)_{i}=\frac{u_{i+\frac{1}{2}}-u_{i-\frac{1}{2}}}{h} \quad, \quad 1 \leq i \leq N
$$

while for boundaries is:

$$
\begin{gathered}
(G u)_{0}=-\frac{8}{3 h} u_{0}+\frac{3}{h} u_{\frac{1}{2}}-\frac{1}{3 h} u_{\frac{3}{2}} \\
(G u)_{N}=\frac{8}{3 h} u_{N}-\frac{3}{h} u_{N-\frac{1}{2}}+\frac{1}{3 h} u_{N-\frac{3}{2}}
\end{gathered}
$$

The expression for mimetic divergence $[\mathbf{D}]$ discretization at block centers is given by the formula:

$$
D_{i+\frac{1}{2}} v=\frac{v_{i+1}-v_{i}}{h}
$$

It is important to notice that equations (2)-(5) are all second order expressions.

\section{Mimetic Crank-Nicolson scheme}

The general form of one dimensional unsteady diffusion equation is represented by:

$$
\frac{\partial u}{\partial t}(\vec{x}, t)=\nabla \cdot(K \nabla u(\vec{x}, t))+F(\vec{x}, t) .
$$

where $K$ is the transfer diffusivity coefficient anf $F$ is the known term source. In order to obtain a well posed problem with an unique and stable solution, we apply a general Robin boundary condition:

$$
\alpha(x) u+\frac{\partial u}{\partial \vec{n}}=f(x, t)
$$

with initial condition:

$$
u(x, 0)=f(x)
$$


We propose a new scheme for the diffusion equation based on Crank-Nicolson scheme, that is obtained by the combination of implicit and explicit difference schemes. The first one takes the form:

$$
\frac{1}{2}[T]\left(U^{n+1 / 2}-U^{n}\right)-[D][K][G] U^{n}=F^{n}
$$

with boundary condition:

$$
[-\alpha[A]-\beta[B G]] u^{n}=f^{n}
$$

where $[T]$ is a diagonal time matrix whose nonzero entries are $\frac{1}{d t}$ with $d t$ the time step. Vectors $U$ and $F$ are temperature and source approximations on the mimetic grid nodes. Similarly explicit scheme is given by the expression:

$$
\frac{1}{2}[T]\left(U^{n+1}-U^{n+1 / 2}\right)-[D][K][G] U^{n+1}=F^{n+1}
$$

with boundary condition:

$$
[-\alpha[A]-\beta[B G]] u^{n+1}=f^{n+1}
$$

where $[A]$ is the corresponding boundary matrix with nonzero entries $\alpha$ and $f$ are approximations on the grid nodes at the boundary. Combining equations (9)-(12) we obtain the mimetic Crank-Nicolson scheme for one dimensional diffusion equation:

$$
\begin{gathered}
{\left[T-\frac{1}{2}([D K G]-\alpha[A]-\beta[B G])\right] u^{n+1}=\left[T+\frac{1}{2}([D K G]-\alpha[A]-\beta[B G])\right] u^{n}+} \\
+\frac{1}{2}\left(F^{n+1}+F^{n}\right)+\frac{1}{2}\left(f^{n+1}+f^{n}\right)
\end{gathered}
$$

It is a system of $(N+2) \times(N+2)$ equations which are listed below.

First equation is given by:

$$
\begin{gathered}
\left(\frac{1}{2} \alpha_{1}+\frac{4}{3 h}\right) u_{0}^{n+1}-\frac{3}{2 h} u_{\frac{1}{2}}^{n+1}+\frac{1}{6 h} u_{\frac{3}{2}}^{n+1}=\left(-\frac{1}{2} \alpha_{1}-\frac{4}{3 h}\right) u_{0}^{n}+ \\
\frac{3}{2 h} u_{\frac{1}{2}}^{n}-\frac{1}{6 h} u_{\frac{3}{2}}^{n}+\frac{1}{2}\left(f_{0}^{n+1}+f_{0}^{n}\right)
\end{gathered}
$$

and represents second order discretization of Robin boundary condition at the node $x_{0}$. By simmetry a similar expression exists for the $x_{N}$ node who is showed in Figure 2.

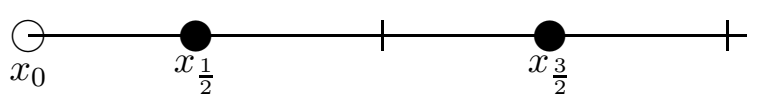

Figure 2: Left boundary node. 
Second equation is:

$$
\begin{gathered}
\left(-\frac{1}{6}-\frac{4}{3 h^{2}}\right) u_{0}^{n+1}+\left(\frac{1}{4 h}+\frac{2}{h^{2}}+\frac{1}{\Delta t}\right) u_{\frac{1}{2}}^{n+1}+\left(-\frac{1}{12 h}-\frac{2}{3 h^{2}}\right) u_{\frac{3}{2}}^{n+1}= \\
\left(\frac{1}{6}+\frac{4}{3 h^{2}}\right) u_{0}^{n}+\left(-\frac{1}{4 h}-\frac{2}{h^{2}}+\frac{1}{\Delta t}\right) u_{\frac{1}{2}}^{n}+\left(\frac{1}{12 h}+\frac{2}{3 h^{2}}\right) u_{\frac{3}{2}}^{n}+\frac{1}{2}\left(F_{\frac{1}{2}}^{n+1}+F_{\frac{1}{2}}^{n}\right)
\end{gathered}
$$

and represents the discretization of unsteady diffusion equation centered in $x_{\frac{1}{2}}$ node. There is a similar formula for the $x_{N-\frac{1}{2}}$ node.

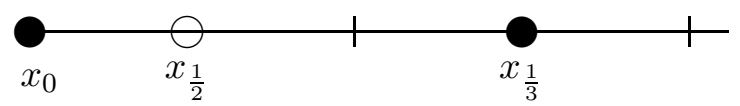

Figure 3: $x_{\frac{1}{2}}$ node.

Third equation is not standard because it contains four terms in its expression. It is centered in the $x_{\frac{3}{2}}$ node.

$$
\begin{gathered}
\frac{1}{6 h} u_{0}^{n+1}+\left(-\frac{1}{4 h}-\frac{1}{2 h^{2}}\right) u_{\frac{1}{2}}^{n+1}+\left(\frac{1}{12 h}+\frac{1}{h^{2}}+\frac{1}{\Delta t}\right) u_{\frac{3}{2}}^{n+1}-\frac{1}{2 h^{2}} u_{\frac{5}{2}}^{n+1}= \\
-\frac{1}{6 h} u_{0}^{n}+\left(\frac{1}{4 h}+\frac{1}{2 h^{2}}\right) u_{\frac{1}{2}}^{n}+\left(-\frac{1}{12 h}-\frac{1}{h^{2}}+\frac{1}{\Delta t}\right) u_{\frac{3}{2}}^{n}+\frac{1}{2 h^{2}} u_{\frac{5}{2}}^{n}+\frac{1}{2}\left(F_{\frac{3}{2}}^{n+1}+F_{\frac{3}{2}}^{n}\right)
\end{gathered}
$$

Because of simmetry a similar expression is given for $x_{N-\frac{5}{2}}$ node (see Figure 4 ).

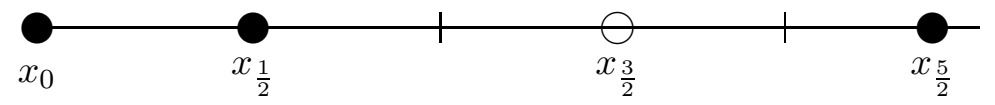

Figure 4: $x_{N-\frac{5}{2}}$ node.

This group of equations correspond to all inner nodes (see Figure 5):

$$
\begin{gathered}
-\frac{1}{2 h^{2}} u m_{i-\frac{1}{2}}^{n+1}+\left(\frac{1}{h^{2}}+\frac{1}{\Delta t}\right) u m_{i+\frac{1}{2}}^{n+1}-\frac{1}{2 h^{2}} u m_{i+\frac{3}{2}}^{n+1}=\frac{1}{2 h^{2}} u m_{i-\frac{1}{2}}^{n}+ \\
+\left(-\frac{1}{h^{2}}+\frac{1}{\Delta t}\right) u m_{i+\frac{1}{2}}^{n}+\frac{1}{2 h^{2}} u m_{i+\frac{3}{2}}^{n}+\frac{1}{2}\left(F_{i+\frac{1}{2}}^{n+1}+F_{i+\frac{1}{2}}^{n}\right)
\end{gathered}
$$

Those are classical expressions of standard finite difference method. 


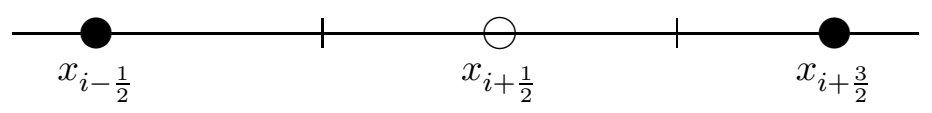

Figure 5: Inner nodes.

\section{Convergence analysis}

Convergence to the exact solution is the most important property of every finite difference method. We will show that mimetic Crank-Nicolson scheme is consistent with diffusion equation and unconditionally stable. Then using Lax theorem we will conclude that new method is convergent.

First step of the proof is the substitution of boundary conditions equations (14) into the equations (15) and (16). After that we obtain the following discretizations:

$$
\begin{gathered}
\left(\frac{-3\left(h^{2}+8\right)}{2 h^{2}\left(3 \alpha_{1}+8\right)}+\frac{h+8}{4 h^{2}}+\frac{1}{\Delta t}\right) u m_{\frac{1}{2}}^{n+1}+\left(\frac{h^{2}+8}{6 h^{2}\left(3 \alpha_{1}+8\right)}-\frac{h+8}{12 h^{2}}\right) u m_{\frac{3}{2}}^{n+1} \\
+\left(\frac{-3\left(h^{2}+8\right)}{2 h^{2}\left(3 \alpha_{1}+8\right)}+\frac{h+8}{4 h^{2}}-\frac{1}{\Delta t}\right) u m_{\frac{1}{2}}^{n}+\left(\frac{h^{2}+8}{6 h^{2}\left(3 \alpha_{1}+8\right)}-\frac{h+8}{12 h^{2}}\right) u m_{\frac{3}{2}}^{n}- \\
-\frac{h^{2}+8}{h\left(3 \alpha_{1}+8\right)} \gamma_{0}=\frac{1}{2}\left(F_{\frac{1}{2}}^{n+1}+F_{\frac{1}{2}}^{n}\right)
\end{gathered}
$$

and

$$
\begin{gathered}
\left(\frac{3}{2 h\left(3 \alpha_{1}+8\right)}-\frac{h+2}{4 h^{2}}\right) u m_{\frac{1}{2}}^{n+1}+\left(\frac{-1}{6 h\left(3 \alpha_{1}+8\right)}+\frac{h+12}{12 h^{2}}+\frac{1}{\Delta t}\right) u m_{\frac{3}{2}}^{n+1}- \\
-\frac{1}{2 h^{2}} u m_{\frac{5}{2}}^{n+1}+\left(\frac{3}{2 h\left(3 \alpha_{1}+8\right)}-\frac{h+2}{4 h^{2}}\right) u m_{\frac{1}{2}}^{n}+\left(\frac{-1}{6 h\left(3 \alpha_{1}+8\right)}+\frac{h+12}{12 h^{2}}-\frac{1}{\Delta t}\right) u m_{\frac{3}{2}}^{n}- \\
-\frac{1}{2 h^{2}} u m_{\frac{5}{2}}^{n}+\frac{\gamma_{0}}{3 \alpha_{1}+8}=\frac{1}{2}\left(F_{\frac{3}{2}}^{n+1}+F_{\frac{3}{2}}^{n}\right)
\end{gathered}
$$

These equations may be written in the next form:

$$
\begin{gathered}
\left(-\frac{1}{6}-\frac{4}{3 h^{2}}\right) u m_{0}^{n+1}+\left(\frac{1}{4 h}+\frac{2}{h^{2}}+\frac{1}{\Delta t}\right) u m_{\frac{1}{2}}^{n+1}+\left(-\frac{1}{12 h}-\frac{2}{3 h^{2}}\right) u m_{\frac{3}{2}}^{n+1}+ \\
+\left(-\frac{1}{6}-\frac{4}{3 h^{2}}\right) u m_{0}^{n}-\left(-\frac{1}{4 h}-\frac{2}{h^{2}}+\frac{1}{\Delta t}\right) u m_{\frac{1}{2}}^{n}-\left(\frac{1}{12 h}+\frac{2}{3 h^{2}}\right) u m_{\frac{3}{2}}^{n}- \\
-\frac{1}{2}\left(F_{\frac{1}{2}}^{n+1}+F_{\frac{1}{2}}^{n}\right)+\frac{h^{2}+8}{h\left(3 \alpha_{1}+8\right)}\left[\left(\frac{1}{2} \alpha_{1}+\frac{4}{3 h}\right) u m_{0}^{n+1}-\frac{3}{2 h} u m_{\frac{1}{2}}^{n+1}+\frac{1}{6 h} u m_{\frac{3}{2}}^{n+1}\right] \\
+\frac{h^{2}+8}{h\left(3 \alpha_{1}+8\right)}\left[\left(-\frac{1}{2} \alpha_{1}-\frac{4}{3 h}\right) u m_{0}^{n}-\frac{3}{2 h} u m_{\frac{1}{2}}^{n}+\frac{1}{6 h} u m_{\frac{3}{2}}^{n}-\gamma_{0}\right]=0
\end{gathered}
$$


and

$$
\begin{gathered}
\frac{1}{6 h}\left(u m_{0}^{n+1}+u m_{0}^{n}\right)+\left(-\frac{1}{4 h}-\frac{1}{2 h^{2}}\right) u m_{\frac{1}{2}}^{n+1}+\left(\frac{1}{12 h}+\frac{1}{h^{2}}+\frac{1}{\Delta t}\right) u m_{\frac{3}{2}}^{n+1}- \\
-\frac{1}{2 h^{2}} u m_{\frac{5}{2}}^{n+1}-\left(\frac{1}{4 h}+\frac{1}{2 h^{2}}\right) u m_{\frac{1}{2}}^{n}-\left(-\frac{1}{12 h}-\frac{1}{h^{2}}+\frac{1}{\Delta t}\right) u m_{\frac{3}{2}}^{n}-\frac{1}{2 h^{2}} u m_{\frac{5}{2}}^{n}- \\
-\frac{1}{2}\left(F_{\frac{3}{2}}^{n+1}+F_{\frac{3}{2}}^{n}\right)-\frac{1}{3 \alpha_{1}+8}\left[\left(\frac{1}{2} \alpha_{1}+\frac{4}{3 h}\right) u m_{0}^{n+1}-\frac{3}{2 h} u m_{\frac{1}{2}}^{n+1}+\frac{1}{6 h} u m_{\frac{3}{2}}^{n+1}\right]- \\
-\frac{1}{3 \alpha_{1}+8}\left[-\left(-\frac{1}{2} \alpha_{1}-\frac{4}{3 h}\right) u m_{0}^{n}-\frac{3}{2 h} f_{\frac{1}{2}}^{n}+\frac{1}{6 h} u m_{\frac{3}{2}}^{n}-\gamma_{0}\right]=0
\end{gathered}
$$

They contain the differential equations and boundary condition approximations in single equation. A Taylor expansion calculation shows that

- for the equation centered on the $x_{\frac{1}{2}}$ node

$$
\frac{\partial u}{\partial t}-\frac{\partial^{2} u}{\partial x^{2}}-\frac{1}{6} h \frac{\partial u^{3}}{\partial x^{3}}+\frac{1}{24} \Delta t^{2} \frac{\partial u^{3}}{\partial t^{3}}
$$

the truncation error is $O(h)+O\left(\Delta t^{2}\right)$.

- For the equation centered on $x_{\frac{3}{2}}$ node

$$
\frac{\partial u}{\partial t}-\frac{\partial^{2} u}{\partial x^{2}}-\frac{5}{48} h^{2} \frac{\partial u^{3}}{\partial x^{3}}+\frac{1}{24} \Delta t^{2} \frac{\partial u^{3}}{\partial t^{3}}
$$

truncation error is $O\left(h^{2}\right)+O\left(\Delta t^{2}\right)$.

- For central equations centered on $x_{i+\frac{1}{2}}$ nodes

$$
\frac{\partial u}{\partial t}-\frac{\partial^{2} u}{\partial x^{2}}-\frac{1}{12} h^{2} \frac{\partial u^{4}}{\partial x^{4}}-\frac{1}{8} \Delta t^{2} \frac{\partial u^{4}}{\partial t^{4}}
$$

truncation errors are $O\left(h^{2}\right)+O\left(\Delta t^{2}\right)$.

Truncation errors for the equation centered on $x_{N-\frac{3}{2}}$ node and for the equation centered on $x_{N-\frac{1}{2}}$ node are the same of the equations (23) y (22).

As a second step we write the associated system using the equations (17), (20) and (21):

$$
(I+r W) u^{n+1}=(I-r W) u^{n}
$$

where $r=\frac{\Delta t}{h^{2}}$ and their eigenvalues are $\mu=\frac{(1-r \lambda)}{(1+r \lambda)}$.

The last step of the proof is to show that $\mu \leq 1$. To obtain this result we have to verify that $\lambda \geq 0$. This is done applying Gershgorin theorem at each equation of system (25), so the spectral radius is less or equal than one and it means that new method is uncoditionally stable and by Lax-Friedrichs theorem is convegent. More details of this proof in [6]. 


\section{Comparative numerical study}

We present one dimensional test problem solved with new mimetic scheme (MIMETIC) and standard finite difference based on ghost points (FD). The equation is:

$$
\frac{\partial u}{\partial t}-\frac{\partial^{2} u}{\partial x^{2}}=-\frac{1}{10} e^{-\frac{t}{10}} \sin (2 \pi x)+4 e^{-\frac{t}{10}} \sin (2 \pi x) \pi^{2}
$$

defined in $(0,1)$ and boundary Robin's contidions:

$$
\begin{aligned}
& u(0)-\frac{\partial u}{\partial x}(0)=-2 \pi e^{-\frac{t}{10}} \\
& u(1)+\frac{\partial u}{\partial x}(1)=2 \pi e^{-\frac{t}{10}}
\end{aligned}
$$

with initial condition:

$$
u(x, 0)=e^{\frac{t}{10}} \sin (2 \pi x)
$$

Equations (23)-(26) form a well posed mathematic problem whith analitic solution:

$$
u(x, 0)=\sin (2 \pi x)
$$

Table 1 shows the magnitud of the relative errors produced by mimetic and finite difference methods. The first ones are smaller than second ones.

\begin{tabular}{c|cc}
\hline \hline Methods & Grid size & Errors \\
\hline \multirow{3}{*}{ MIMETIC } & 30 & $5.5000 \times 10^{-3}$ \\
& 60 & $1.4000 \times 10^{-3}$ \\
& 100 & $5.0298 \times 10^{-4}$ \\
\hline \multirow{3}{*}{ FD } & 30 & $1.0980 \times 10^{-1}$ \\
& 60 & $5.3500 \times 10^{-2}$ \\
& 100 & $3.1800 \times 10^{-2}$ \\
\hline \hline
\end{tabular}

Table 1: Relative errors in infinity norm.

The convergence rates are reported in table 2 .

\begin{tabular}{c|cc}
\hline \hline Methods & Grid size & Orders \\
\hline \multirow{3}{*}{ MIMETIC } & 30 & 2.1108 \\
& 60 & 2.0445 \\
& 100 & 2.0202 \\
\hline \multirow{3}{*}{ FD } & 30 & 1.0758 \\
& 60 & 1.0620 \\
& 100 & 1.0503 \\
\hline \hline
\end{tabular}

Table 2: Convergence orders. 


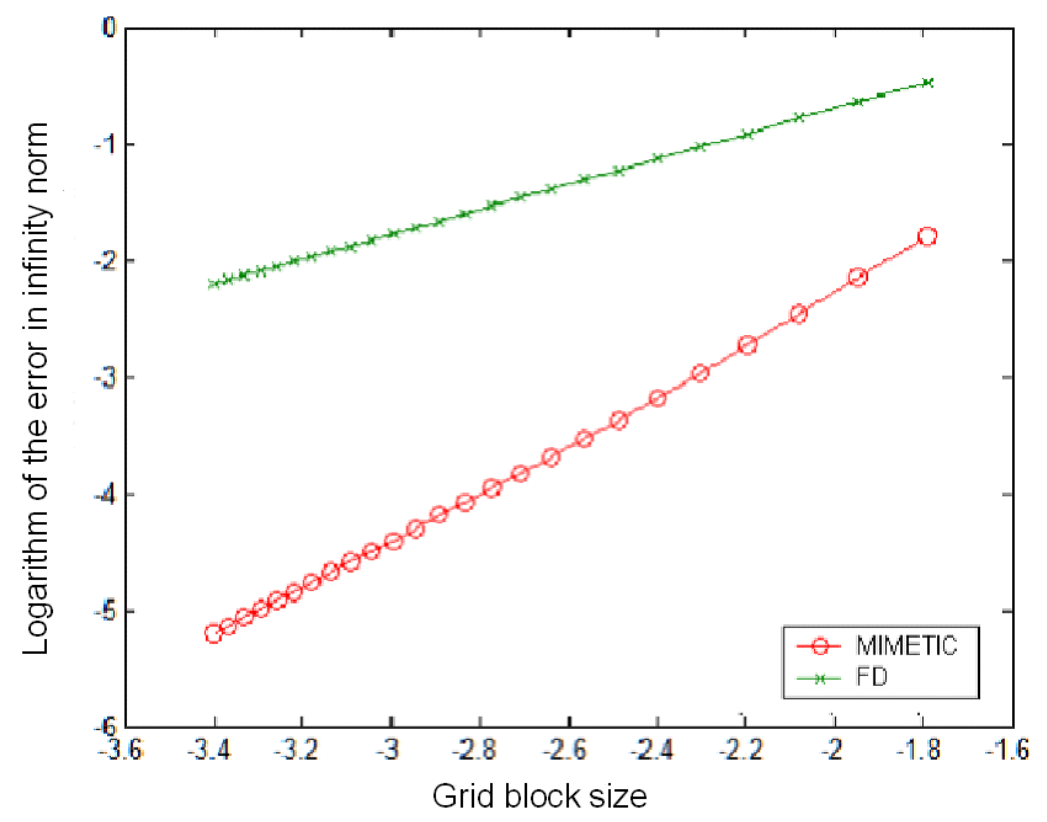

Figure 6: Errors in modulus maximun norm.

Graphical representation of errors computed in modulus maximum norm is displayed in Figure 6.

Here the lower is a line better is the approximation. Observe that blue line associated with mimetic scheme is below green line associated with finite difference method, this remark that new scheme is better than the other method.

\section{Conclusions and discussion}

A new mimetic scheme to approximate the solution of diffusion equation has been presented. We show that new method is consistent, unconditionally stable and convergent. Comparative numerical study shows that mimetic method produces smaller errors than those obtained with standard finite difference and second order convergence rates. This could be attributed to the use of ghost points in FD scheme. Computer implementation of new method is not harder than FD scheme and the high quality of the approximation evidences that mimetic method is a good alternative in the solution of diffusion problems. 


\section{References}

[1] Castillo, J.E.; Grone, R.D. (2003) "A matrix analysis approach to higher-order approximations for divergence and gradients satisfying a global conservation law", SIAM Journal on Matrix Analysis and Applications 25(1): 128-142.

[2] Freites-Villegas, M.; Guevara-Jordan, J.M.; Rojas, O.; Castillo, J.E.; Rojas S. (2004) "A mimetic finite difference scheme for solving the steady state diffusion equation with singular sources", VII International Congress of Numerical Methods in Engineering and Science, San Cristobal, Venezuela.

[3] Castillo, J.E.; Yasuda, M. (2005) "Linear system arising for second order mimetic divergence and gradient operators", Journal of Mathematical Modeling and Algorithm 4(1): $67-82$.

[4] Shashkov, M. (1996) "Conservative Finite-Differences Methods on General Grids", Symbolic and Numerical Computation Series, CRC Press, Boca Raton FL.

[5] Guevara-Jordan, J.M.; Rojas, S.; Freites-Villegas, M.; Castillo J.E. (2007) "Convergence of a mimetic finite difference method for static diffusion equation", Advances in Difference Equations, Volume 2007, Article ID 12303, 12 pages.

[6] Mannarino, I. (2007) "Un método mimético de diferencias finitas para la ecuación no estática de difusión", Master thesis, Universidad Central de Venezuela, Caracas.

[7] Mannarino, I.; Quintana, Y.; Guevara-Jordan, J.M. (2007) "A numerical study of mimetic scheme for the unsteady heat equation", submitted to FACYT Review (Faraute). 